\title{
The evolution of Dscam genes across the arthropods
}

\author{
Sophie AO Armitage ${ }^{1 *+}$, Rebecca Y Freiburg ${ }^{1+}$, Joachim Kurtz ${ }^{1}$ and Ignacio G Bravo ${ }^{1,2}$
}

\begin{abstract}
Background: One way of creating phenotypic diversity is through alternative splicing of precursor mRNAs. A gene that has evolved a hypervariable form is Down syndrome cell adhesion molecule (Dscam-hv), which in Drosophila melanogaster can produce thousands of isoforms via mutually exclusive alternative splicing. The extracellular region of this protein is encoded by three variable exon clusters, each containing multiple exon variants. The protein is vital for neuronal wiring where the extreme variability at the somatic level is required for axonal guidance, and it plays a role in immunity where the variability has been hypothesised to relate to recognition of different antigens. Dscam-hv has been found across the Pancrustacea. Additionally, three paralogous non-hypervariable Dscam-like genes have also been described for D. melanogaster. Here we took a bioinformatics approach, building profile Hidden Markov Models to search across species for putative orthologs to the Dscam genes and for hypervariable alternatively spliced exons, and inferring the phylogenetic relationships among them. Our aims were to examine whether Dscam orthologs exist outside the Bilateria, whether the origin of Dscam-hv could lie outside the Pancrustacea, when the Dscam-like orthologs arose, how many alternatively spliced exons of each exon cluster were present in the most common recent ancestor, and how these clusters evolved.

Results: Our results suggest that the origin of Dscam genes may lie after the split between the Cnidaria and the Bilateria and supports the hypothesis that Dscam-hv originated in the common ancestor of the Pancrustacea. Our phylogeny of Dscam gene family members shows six well-supported clades: five containing Dscam-like genes and one containing all the Dscam-hv genes, a seventh clade contains arachnid putative Dscam genes. Furthermore, the exon clusters appear to have experienced different evolutionary histories.

Conclusions: Dscam genes have undergone independent duplication events in the insects and in an arachnid genome, which adds to the more well-known tandem duplications that have taken place within Dscam-hv genes. Therefore, two forms of gene expansion seem to be active within this gene family. The evolutionary history of this dynamic gene family will be further unfolded as genomes of species from more disparate groups become available.
\end{abstract}

Keywords: Alternative splicing, Dscam-like, Gene duplication, Ortholog, Co-ortholog, Paralog

\section{Background}

The biological complexity of an organism does not appear to correlate with the number of protein-coding genes in that organism. This is exemplified by comparing the human with the nematode Caenorhabditis elegans genome, where roughly similar numbers of genes $(20,000-$ 25,000 and 19,000, respectively) result in organisms with

\footnotetext{
*Correspondence: sophie.armitage@uni-muenster.de

† Contributed equally

'Institute for Evolution and Biodiversity, University of Münster, Hüfferstrasse 1, 48149 Münster, Germany

Full list of author information is available at the end of the article
}

startlingly contrasting complexity [1,2]. A pervasive contributor that may in part account for this is alternative splicing of precursor messenger RNA, creating transcriptomic and proteomic diversity [3-5]. A particularly remarkable example is the Down syndrome cell adhesion molecule (Dscam) gene in Drosophila melanogaster, which can potentially generate $38,016 \mathrm{mRNA}$ isoforms via mutually exclusive alternative splicing of exons that encode the ecto- and transmembrane domains [6]; this number increases to 152,064 when one considers independent alternative splicing of exons within the endodomain ([7,8]; Figure 1). The latter figure accounts for more than eleven
C Biomed Central

(ㄷ) 2012 Armitage et al; licensee BioMed Central Ltd. This is an Open Access article distributed under the terms of the Creative Commons Attribution License (http://creativecommons.org/licenses/by/2.0), which permits unrestricted use, distribution, and reproduction in any medium, provided the original work is properly cited. 
times the number of genes in D. melanogaster's genome [9]. Mutually exclusive alternative splicing occurs for ectodomain encoding exons 4, 6 and 9 and transmembrane exon 17 ([6]; Figure 1A). One of twelve exon 4 alternatives, one of 48 exon 6 alternatives, one of 33 exon 9 alternatives and one of two exon 17 alternatives are present in each mRNA ([6]; Figure 1B), and endodomain exons 19 and 23 can be contained or lacking [8]. Because of the extreme number of isoforms possible, Dscam has more recently been referred to as hypervariable, $D s c a m-h v$ [10], a nomenclature that we also adopt in this paper, but it is noteworthy that earlier papers do not use the $-h v$ suffix (e.g. [6]).

The Dscam- $h v$ gene is a member of the Immunoglobulin (Ig) superfamily [11]. In D. melanogaster it plays an essential role in neuronal wiring: it is an axon guidance receptor [6] and it ensures that olfactory receptor neurons synapse in the correct target [12]. The importance of isoform diversity in neuronal wiring and self-recognition has since been uncovered [13-16]. In 2005 Watson and co-workers [17] discovered that depletion of Dscam$h v$ impairs the ability of $D$. melanogaster haemocytes to phagocytose bacteria, spurring research into the immunological role that it could play in Drosophila and other pancrustaceans (i.e., the clade consisting of crustaceans and insects) [17-20]. It has been hypothesised that the large diversity of Dscam- $h v$ isoforms could provide specificity for antigen recognition [21-23], however evidence to support this hypothesis is limited. Dscam- $h v$ is particularly interesting because of its versatility due to the extreme diversity that it encodes, and also because the nervous and immune systems may exert different selection pressures on this gene [24].

The protein domain structure of Dscam-hv in D. melanogaster [6] consists of ten Ig domains: the alternatively spliced exon 4 lies within Ig2, exon 6 within Ig3, and exon 9 comprises the whole of Ig7 (Figure 1C). There are also six fibronectin type III (FN) repeats, a transmembrane domain and a C-terminal cytoplasmic tail [6]. Orthologs of the Dscam-hv gene have been found in other pancrustaceans (Figure 2), including several insect $[17,25]$ and crustacean species $[10,20,26,27]$, and all have the same ectodomain protein structure as D. melanogaster. However, there is considerable variation in the total number of alternatively spliced exons in the Dscam- $h v$ genes across the Pancrustacea $[10,17,26,28]$, and there is also variation in the number of conserved exons. Therefore for simplicity, hereafter we will refer to the hypervariable exons using domain numbering as these are likely to be more conserved than exon number. The question then arises of whether one could reconstruct the history of duplications and deletions that have shaped Dscam-hv evolution, and infer the ancestral set of alternatively spliced exons that were present at the base of the arthropods, pancrustaceans and insects. Also, of particular importance to the question of the ubiquity of Dscam-hv, it remains unclear when alternative splicing as found in Dscam- $h v$ arose [11]. It has been assumed that Dscam- $h v$ was already present in the genome of the common ancestor of the Pancrustacea but it is not known whether it was already present in the

A

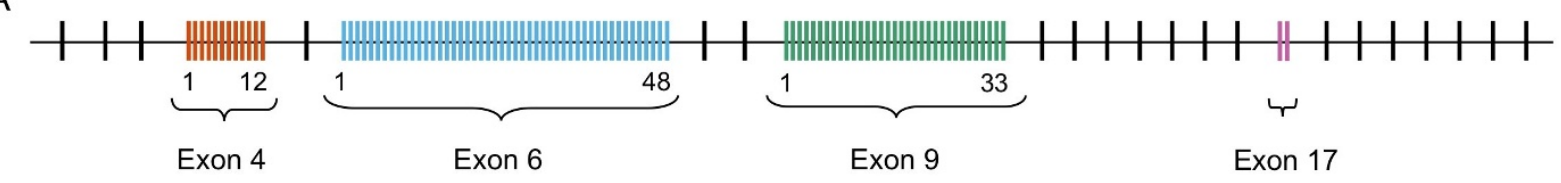

B

\begin{tabular}{|l|l|l|l|l|l|l|l|l|l|l|l|l|l|l|l|l|l|l|l|l|l|l|l|}
\hline 1 & 2 & 3 & 4 & 5 & 6 & 7 & 8 & 9 & 10 & 11 & 12 & 13 & 14 & 15 & 16 & 17 & 18 & 19 & 20 & 21 & 22 & 23 & 24 \\
\hline
\end{tabular}

C

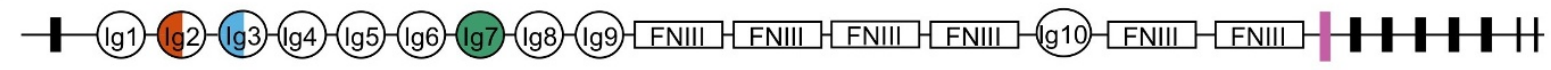

Figure 1 (A) Dscam-hv genomic DNA for Drosophila melanogaster. The gene consists of 20 constant exons (shown as black lines), mutually exclusive alternative splicing occurs for exons 4 (red lines), 6 (blue lines), 9 (green lines) and 17 (purple lines); one of 12 exon 4 alternatives, one of 48 exon 6 alternatives, one of 33 exon 9 alternatives and one of two exon 17 alternatives are present in each mRNA. This enables the vast number of $12 \times 48 \times 33 \times 2=38,016$ potential splice variants. (B) Dscam-hv mRNA. Constant exons are shown as white boxes. Exons that undergo mutually exclusive alternative splicing follow the same colour scheme as for the genomic structure. Endodomain exons 19 and 23 can be contained or lacking [8], which increases the number of potential isoforms to $4 \times 38,016=152,064$. (C) Dscam-hv protein structure for $D$. melanogaster. The alternatively spliced exons encode the N-terminal half of Ig2 (exon 4 in Drosophila); the N-terminal half of Ig3 (exon 6 in Drosophila), all of Ig7 (exon 9 in Drosophila), and the transmembrane domain (Exon 17 in Drosophila (figure after [6]). 


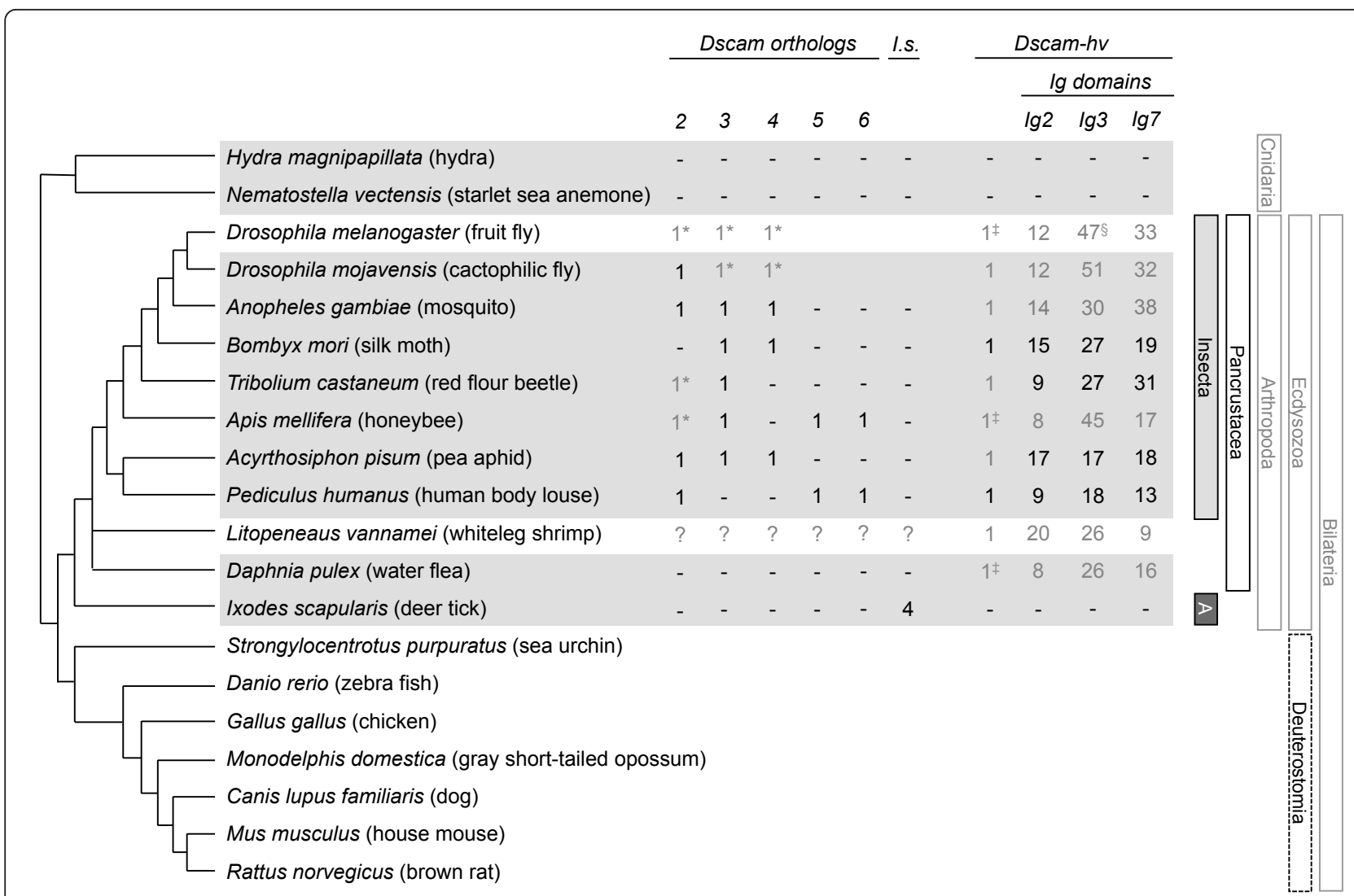

Figure 2 Phylogenetic relationships between the species included in this study. The presence and number of Dscam orthologs and coorthologs (I. scapularis, I.s.), Dscam-hv orthologs, and putative alternatively spliced exons within Immunoglobulins 2, 3 and 7, are indicated for each species. Grey horizontal boxes highlight the species for which our study adds some information: more specifically, black numbers show putative genes found or annotated in this study and black dashes show that we did not find Dscam orthologs/paralogs for that species. Grey numbers show the Dscam genes and Dscam-hv that were annotated or described prior to this study, although the number of Ig exon variants shown are those predicted by our HMMs. The genome of L. vannamei is not sequenced so the grey question marks indicate that we could not search for Dscam-like genes in this species. * indicates genes which were used to build HMMs to search for Dscam orthologs, and $\neq$ indicates genes which were used to build the Dscam-hv HMMs. § For reasoning why this is 47 and not 48, please see Additional file 2. L. vannamei alternatively spliced exon numbers are conservative estimates [26]. Vertical bars indicate the main taxa of interest in this study, where A stands for Arachnida. Species relationships from [29-35].

common ancestor of the arthropods. To date the most closely related species to the pancrustaceans that has been examined for Dscam-hv is the nematode C. elegans, although evidence of multiple exon variants was not found [28], and Brites and co-workers [10] subsequently suggested that it lacks Dscam altogether. Similarly more distantly related taxa within Protostomia, such as a platyhelminth and a mollusc, appear not to have Dscam$h v[10,11]$.

In addition to the hypervariable Dscam- $h v$ gene, three non-hypervariable Dscam genes, so-called Dscam-like genes have been described in D. melanogaster [36]. One such gene, Dscam2, has two alternatively spliced exons in Ig7 [37], whereas Dscam3 and Dscam4 have only one isoform each. Dscam 2 and Dscam 3 have also been ascribed neuronal roles $[37,38]$. The ancestor to at least one of the Dscam-like genes was already present in the common ancestor of the holometabolous insects (Figure 2), because Dscam-like genes have been predicted in the genomes of the honeybee Apis mellifera (AbsCAM [39]), the yellow fever mosquito Aedes aegypti (used in the Dscam gene phylogeny presented in [10]) and the red flour beetle Tribolium castaneum (NCBI gene prediction XP_967655.2). A first approximation of the evolution of the insect Dscam gene family including the aforementioned genes was described by Brites and co-workers [10], however, it is unclear whether all of these species contain orthologs to all three Dscam-like genes as found in D. melanogaster, and therefore also at what point the Dscam gene family diversified: were the three Dscam-like genes already present in the common ancestor of the holometabolous insects, of all insects, or of the pancrustaceans?

Dscam orthologs are also present in deuterostomes. Unlike its pancrustacean counterpart, vertebrate DSCAM 
has only two predicted isoforms [40]. DSCAM also plays a fundamental role in, e.g., axon guidance [41] and selfavoidance [42], but as of yet no immune function has been suggested. A further vertebrate DSCAM homolog has also been identified (DSCAML1 [43]). Phylogenetic inference shows that the Dscam/DSCAM genes in protostomes and deuterostomes cluster separately; it follows that separate duplication events may have occurred leading to the independent expansion of the Dscam/DSCAM families in both lineages [10]. It seems that taxa quite distant from the bilateria (i.e., a plant and a yeast species) do not contain Dscam orthologs [28], however, it is unknown whether Dscam exists in a more closely related group to the Bilateria, e.g., the Cnidaria.

In this paper we take a bioinformatics approach, constructing Hidden Markov Model profiles (HMMs $[44,45])$ to search within the genomes of species for putative Dscam genes and hypervariable alternatively spliced exons and then reconstruct phylogenetic relationships between these genes and exons, to examine the evolutionary history of the Dscam gene family and the evolution of the three extracellularly expressed alternatively spliced exon clusters. Specifically we examine i) whether Dscam orthologs exist outside the Bilateria, if this is the case, it would suggest the origin of the Dscam gene family lies further back than previously believed, ii) whether the origin of Dscam-hv could lie outside the Pancrustacea, iii) when the Dscam-like orthologs arose, iv) how many alternatively spliced exons of each exon cluster were present at the base of the Arthropoda, Pancrustacea and the Insecta and v) whether the three exon clusters evolved similarly.

\section{Methods}

For a general overview of the workflow followed, and extra methodological details, please see Additional files 1 and 2 , respectively.

\section{Hidden Markov models}

The complex exon structure of Dscam-like and Dscam-hv genes (Figure 1), and the fact that the exon structure is highly variable across Dscam orthologs, makes an ortholog search via the reciprocal best blast hit or the reciprocal smallest distance method [46] difficult. This is because these algorithms use alignment methods to search for orthologs that cannot incorporate the large introns contained in the Dscam genes, especially as they are interspersed with short exons. We therefore used profile Hidden Markov Models (HMMs) [44,45,47,48] (Additional files 1 and 2) as our technique to search for homologous Dscam sequences. HMMs are powerful tools often used for annotating sequences, for example domains in a protein sequence, or exons and introns in a nucletide sequence. An HMM is based on a probabilistic model, which in a biological context is usually provided by a multiple sequence alignment. From this model, probabilities are inferred for how likely it is that a certain observation in another sequence is made because the sequence fits the model, or how likely it is that the observation is made despite the sequence not fitting the model. We used HMMs to annotate protein sequences, both full putative Dscam proteins and individual exons, based on an alignment of already known protein sequences for the exon or protein.

\section{Genomes searched and sequences used for tree constructions}

i) To examine whether Dscam homologs exist outside the Bilateria we included two cnidarians, Hydra magnipapillata (Figure 2; genome version 1.0 [49]) and the starlet sea anemone Nematostella vectensis (Figure 2; genome version 1.0 [50]).

ii) To test whether Dscam- $h v$ exists outside of the Pancrustacea we searched the only arachnid (draft version assembly IscaW1, gene set ID IscaW1.1) genome available to date, the deer tick, Ixodes scapularis [51].

iii) To estimate when the Dscam-like orthologs arose we searched for Dscam-like genes in I. scapularis, as well as within nine pancrustaceans: the water flea, Daphnia pulex (genome version 1.0 [52]), six representative holometabolous insects, the mosquito Anopheles gambiae (genome version 3.4 [53]), Apis mellifera (genome version 4.0 [54]), the silk moth Bombyx mori (genome version 2.0 [55]), the flies $D$. melanogaster (genome version 5.0 [9]) and D. mojavensis (genome version 1.0 [56]) Tribolium castaneum (genome version 2.0 [57]) and two hemimetabolous insects, the pea aphid Acyrthosiphon pisum (genome version 1.0 [58]) and the human body louse Pediculus humanus (genome version 1.0 [59]). To infer the relationships between Dscam-hv and Dscam-like genes we included Dscam- $h v$ from the whiteleg shrimp Litopenaeus vannamei [26], as well as DSCAM sequences from the California purple sea urchin Strongylocentrotus purpuratus, the zebra fish Danio rerio, the chicken Gallus gallus, the gray short-tailed opossum Monodelphis domestica and the house mouse Mus musculus; and DSCAML1 sequences from the dog Canis lupus familiaris and the brown rat Rattus norvegicus.

iv) and v) To examine the number of alternatively spliced exons at the base of the Pancrustacea and the evolutionary histories of the three exon clusters, we searched the previously annotated Dscam- $h v$ genes as well as our newly annotated Dscam- $h v$ genes from the above nine pancrustaceans and the putative Dscam genes identified for I. scapularis for alternatively spliced exons and then used these in our phylogenetic analyses. 


\section{Identification of, and protein predictions for, Dscam-like orthologs}

To identify Dscam-like orthologs we constructed HMMs from seven already available Dscam-like sequences. From D. melanogaster this included Dscam2 (see Additional file 3 for the NCBI accession number of this and the following sequences), Dscam3 and Dscam4. Two genes from D. mojavensis that are orthologous to Dscam3 and Dscam4, and two further Dscam-like orthologs from T. castaneum and A. mellifera. The protein sequences were aligned using MUSCLE [60]. The exon structure of these genes is variable between species, therefore to construct the HMMs (using HMMER 2.3.2; http://hmmer. org/[44]) we identified ten highly conserved regions of the alignment (Additional file 4) that did not cross exon boundaries in any of the D. melanogaster Dscam2, Dscam3 or Dscam4 genes (Additional file 5). We translated the genomes of the arthropods A. gambiae, A. mellifera, A. pisum, B. mori, D. pulex, D. mojavensis, I. scapularis, P. humanus and T. castaneum as well as the cnidarians, H. magnipapillata and N. vectensis into six reading frames and the resulting protein sequences were searched for matches to the ten HMMs using the hmmpfam algorithm. We took a conservative approach and considered the prediction as a putative Dscam ortho$\log$ when six of the ten HMMs matched the scaffold in the correct order (for rationale see Additional files 4, 6, 7 and 8 ) and each HMM match reached an e-value of 0.001 or less. Furthermore, we did not predict the domain structures, thus some domains may not be present for some of the Dscam-like predictions. To obtain the complete sequence of the predicted proteins, the Fgenesh + algorithm [61] was used on the identified scaffolds, using the most closely related (species-wise) available Dscam-like sequence as an orthologous sequence. Every predicted protein sequence was aligned to the already-annotated Dscam-like sequences and manually checked for quality.

As a positive control and to explain the underlying rationale for only accepting putative Dscam-like orthologs if six of the ten HMMs (Additional files 4 and 6) matched, we constructed another set of HMMs without D. mojavensis Dscam-like genes and ran these HMMs on the translated D. mojavensis genome. Dscam-hv and three Dscam-like genes were found with all ten HMMS showing significant hits (Additional file 7). Using this data, we started to find non-Dscam genes when four of the HMMS matched our criteria (Additional file 7), but for five HMMs and above we only retrieved the three Dscam-like genes and Dscam-hv, and from Additional file 7 is apparent that five HMMs is in the plateau phase. We therefore suggest that we are more likely to have have false negatives rather than false positives as a result of using six HMMs and above as the cut-off value (see
Additional file 8 for HMM results for all arthropods). As a negative control we created a random nucleotide 'genome'; this contained 55,000 scaffolds each consisting of 30,000 nucleotides (an order of magnitude larger than the D. melanogaster genome) translated into all six reading frames. When we searched this data set with the HMMs, it never yielded more than one hit per scaffold with an e-value of less than 0.01 .

\section{Identification and annotation of Dscam-hv genes}

To build the HMMs for the Dscam- $h v$ genes, we aligned the complete Dscam- $h v$ protein sequences from A. mellifera (see Additional file 3 for the NCBI accession number of this and the following sequences), D. melanogaster and D. pulex using MUSCLE and annotated all exon borders. The global alignment was divided into 37 smaller alignments, which covered nearly the whole gene, whilst ensuring that none of the HMMs included exon borders; we then used HMMER to construct the protein HMMs from these alignments (Additional file 9). We used these HMMs to annotate previously incompletely annotated $D s c a m-h v$ exons from sequences that were already available from the literature for A. gambiae, B. mori and T. castaneum. The nucleotide sequences were translated into three reading frames and each HMM searched for the best two hits within these three reading frames. The best hits were then assembled into the full mRNA and protein sequences. For the remaining species where Dscam-hv had not been already fully annotated (A. pisum, I. scapularis and $P$. humanus), the whole genomes were translated into all six reading frames. The $37 \mathrm{HMMs}$ were used to search the translated genomes and candidate genes proposed when more than 10 of these HMMs matched well (an evalue lower than 0.01 because these HMMs were longer than for the Dscam-like genes). These scaffolds were treated in the same way as the already identified sequences from A. gambiae, B. mori, and T. castaneum. For one species, $A$. pisum, this method was able to identify two scaffolds, one containing the first third of Dscam- $h v$ and the other that overlapped this scaffold and contained the remaining two thirds. The two scaffolds were manually edited together, resulting in the full gene sequence. We also ran the HMMs over the translated $H$. magnipapillata and $N$. vectensis genomes.

\section{Identification and annotation of Dscam-hv hypervariable exons}

To identify and annotate the hypervariable exons in the Dscam-hv orthologs of A. gambiae, A. pisum, B. mori, P. humanus and T. castaneum, and to search for hypervariable exons in I. scapularis, HMMs of the previously annotated exon variants of $D$. melanogaster, A. mellifera, and D. pulex were constructed (see Additional files 2, 10, 11 and 12). Not all hypervariable exons were used to 
build the HMMs, as using many exons that are closely related could result in over-representation of these exons, and may have lead to non-detection of exon variants that are evolutionarily further away. Therefore, approximate trees (RAxML, 100 rapid bootstraps with GTR $+\Gamma 4$ ) were built from all available sequences for each of Ig2, Ig3 and Ig7, and only one representative exon from each clade was used for the HMMs. As a positive control, these HMMs were tested against the intronic regions of species in which these exons have already been annotated and all previously annotated exon variants could be found. We focused on the exons that correspond to extracellular domains of the protein, i.e. 4, 6 and 9. Therefore the intronic regions between the exons homologous to D. melanogaster exons 3 and 5, 5 and 7, and 8 and 10 of the species mentioned above, were translated into six reading frames and searched using the HMMs. All six reading frames were used so that potential inversion events could also be detected. After inspecting the distribution of e-values among the matches (Additional file 13), a cut-off of 0.001 was chosen for the exons to be included in the rest of the analyses. Nucleotide sequences of putatively alternatively spliced exons were then retrieved from the intronic sequences to use for the phylogenies.

\section{Phylogeny of the Dscam gene family}

An alignment of all known and newly-identified Dscamlike orthologs as well as all Dscam-hv genes and the Deuterostoma DSCAM orthologs was created using several methods. First, a complete protein alignment of all 44 sequences was created using MUSCLE. All Ig2, Ig3, and Ig7 orthologous regions were removed from the alignment. The resulting 3,096 amino acid positions-long alignment was then shortened to $47 \%$ of its original length using Gblocks, allowing for gap positions [62] in order to allow for a more accurate deep phylogeny without alignment mismatches obscuring these deep nodes, and to break down artifacts such as long branch attraction [63]. The final alignment encompassed 44 sequences and spanned 1467 positions, comprising of 1420 alignment patterns and $14.9 \%$ gaps and undetermined positions (Additional file 14). The LG protein substitution matrix [64] was identified as the best-suited evolutionary model for our data set using ProtTest [65]. From this alignment, phylogenetic inference was performed on a maximum likelihood framework using RAxML v7.2.8 [66] with the LG $+\Gamma 4$ model and computing 1,000 bootstrap replicates starting with a random tree. Bayesian phylogenetic inference was performed in parallel using PhyloBayes 3.3 [67] and LG $+\Gamma 4$ and the CAT approximation for profile mixture. Three independent Monte Carlo Markov chains were launched, allowed to reach stationary state, checked for convergence among them and the posterior distribution sampled every 100 values to collect 1,000 data points. Rogue taxa, as defined by Pattengale and co-workers [68] were identified with RAxMLv7.2.8 using the -f R option.

Both maximum likelihood and Bayesian approaches recovered sequences from arthropods and deuterostomes as being respectively monophyletic. Arthropod sequences clustered into seven well-supported clades, although their relative phylogenetic relationships could not be inferred with confidence. To test different alternative hypotheses for the evolution of the Dscam gene family relationships we constructed alternative topologies and performed the Shimodaira-Hasegawa test on them (SH-test [69]) (Additional file 15), as implemented in RAxMLv7.2.8. The SHtest allows one to compare different trees for a given alignment under a maximum likelihood framework, and to identify alternative topologies that may also be acceptable descriptions for the phylogenetic relationships among clades. The preferred tree was chosen by testing the equally good gene trees identified against the known evolutionary relationships among the corresponding organisms.

Time inference for the Dscam gene family was performed with PhyloBayes, fixing the topological relationships among clades to follow the best tree and the preferred tree (Additional file 15), using $L G+\Gamma 4$ and the CAT approximation for profile mixture, a log normal model [70] for the relaxed clock, and a birth-death prior. The following species divergence times were used as calibrations in the clades in square brackets: fruit fly mosquito, 238.5 - 295.4 Ma [Dscam2/Dscam3/Dscam4/ Dscam-hv]; fruit fly - bee, 238.5 - 307.2 Ma [Dscam2/ Dscam3]; human - zebrafish, 416.1 - 421.8 Ma [DSCAM]; human - cow, 95.3 - $113 \mathrm{Ma}$ [DSCAM1] (divergence dates from [29]). Three Monte Carlo Markov chains were constructed and tested for convergence.

\section{Phylogeny of Dscam-hv exons}

To construct the phylogenies of Dscam- $h v$ exon clusters, the protein sequences of all putative exons found for that cluster were aligned together with one representative exon of an I. scapularis gene, which was identified in the preferred Dscam tree to serve as an outgroup for the arthropod genes. Only one exon sequence of L. vannamei was included for each of the three exon clusters, as the rest of the exon sequences were not yet publicly available. The protein sequences were aligned using MUSCLE, manually checked for quality and then back-translated into codon alignments using PAL2NAL [71]. At the nucleotide level the final alignments encompassed respectively for the Ig2, Ig3 and Ig7 exons, 106, 290 and 219 sequences; 197, 147 and 328 alignment patterns; and $22 \%, 18 \%$ and $21 \%$ gaps and completely undetermined positions (Additional files 16, 17 and18). Phylogenetic 
inference was performed with RAxML v7.2.6 at the nucleotide level using GTR $+\Gamma 4$, without partitions and also introducing three partitions corresponding to the three codon positions. Two independent runs with 5,000 bootstrap cycles were calculated, starting with a random tree. Bayesian phylogenetic inference was also performed on the same alignment with PhyloBayes at the nucleotide level using GTR $+\Gamma 4$ and the CAT approximation for profile mixture. Similar methods were used to infer relationships between the $D$, melanogaster and the $D$. mojavensis Dscam-hv exons only. In this case, at the nucleotide level the final alignments encompassed respectively for the Ig2, Ig3 and Ig7 exons, 25, 99 and 66 sequences; 148, 128 and 296 alignment patterns; and $6.8 \%, 8.9 \%$ and $0.6 \%$ gaps and completely undetermined positions (Additional files 19, 20 and 21).

\section{Results and discussion}

We have identified novel putative members of the Dscam gene family across the Pancrustacea. These fell into six well-supported insect gene clades: five Dscam-like and one containing the Dscam- $h v$ cluster. A seventh wellsupported clade included the four Dscam-related genes present in the genome of the arachnid I. scapularis, each containing a maximum of only one Ig2, one Ig3 and one Ig7 variant. These findings suggest that the Dscam genes duplicated independently in the insects and an arachnid genome, which adds to the two independent DSCAM gene copies found in vertebrate genomes. Similarly to tropomyosin genes [72], a second form of gene expansion, tandem duplication, is also highly active in this gene family, and is thought to have resulted in numerous alternatively spliced exon variants. We also identified alternatively spliced exons within Ig2, Ig3 and Ig7 from various insect species, but the short sequence length and the limited amount of phylogenetic signal of the putative exons identified, precluded the reconstruction of deep relationships between the exon variants of all arthropods.

\section{Identification of, and protein predictions for, members of the Dscam family}

Using probabilistic models (profile HMMs) derived from multiple protein sequence alignments of Dscam-like and of Dscam-hv, we searched across taxa for potential members of the Dscam family. Using our Dscam-like HMMs we were unable to detect any Dscam-like orthologs in either of the two cnidarian genomes (N. vectensis and $H$. magnipapillata; Figure 2; we found similar negative results for the Dscam-hv HMMs). This supports the hypothesis that the ancestor of the Dscam gene family appeared after the emergence of the Bilateria. However, it is possible that we did not find any orthologs because of gaps in the genome sequences, so it would be informative to re-run this search when genomes of better quality are available. It is worth noting that we have chosen conservative HMM cut-offs (Additional files 7 and 8), and we are aware that this may lead to negative results and may have excluded true orthologs if their phylogenetic signal was too weak or had been eroded.

Across the arthropod genomes searched, we found 20 putative Dscam-like genes, four of which were in I. scapularis (Figure 2; Additional file 6). All insect genomes contained between two and four putative Dscam-like genes. Similarly to Brites and co-workers [10], we could not find any putative genes closely related to Dscam-like in the $D$. pulex genome, although the authors did find two regions with homology to the Dscam-like genes that contained a different domain organisation [10] (see also unpublished data cited in [73]). We did not find any putative Dscam-hv genes in the tick genome, which supports the hypothesis that Dscam-hv, as found in the insects, whiteleg shrimp, and water flea, originated at the base of the Pancrustacea. To test this hypothesis further, it would be essential to scan more non-pancrustacean arthropod genomes when they become available, and also to examine the tick genome when it is fully assembled. We did, however, identify $D s c a m-h v$ for all of our pancrustacean species (Figure 2). No duplicated Dscam-hv genes were found in any of the genomes searched.

\section{Phylogeny of the Dscam gene family}

With the newly identified sequences we aimed to reconstruct the phylogenetic relationships among the Dscam- $h v$ and Dscam-like genes in arthropods, using deuterostome Dscam genes as an outgroup (Figure 2), and to incorporate time estimates in the Bayesian calculations by introducing priors with information from the fossil record. Both maximum likelihood and Bayesian phylogenetic reconstructions (Additional files 22 and 23), suggested that all of the $D s c a m-h v$ genes form one clade. Both methods also identified five other well-supported insect clades of Dscam-like genes, three of which clustered around one of the three D. melanogaster Dscam-like genes, and which we therefore named as Dscam2, Dscam 3 and Dscam4 according to which of the three D. melanogaster genes they were orthologous to (Figure 2, Additional files 6, 22 and 23). The sequences from the I. scapularis genome clustered together to form one clade, and are therefore co-orthologs [74] with respect to the rest of Dscam-hv and Dscam-like clusters. Although the monophyly of each of the aforementioned seven large clades was clear, the phylogenetic position of certain individual taxa within the corresponding clade could not be clearly resolved, as several alternative branching positions could be identified to attain more than 0.95 posterior probability [68]. Such rogue taxa included co-orthologs a and c among the Dscam genes in I. scapularis, sequences from T. castaneum, B. mori, $A$. pisum and A. mellifera among the Dscam- $h v$ genes, and 
sequences from $T$. castaneum and $A$. mellifera among the Dscam 2 genes. In these instances, the inferred phylogenetic relationships among genes did not always match the evolutionary relationships among the corresponding organisms (Figures 2 \&3). Problematic placements included B. mori (Figure 3, Dscam-hv and Dscam3 clades), where the predicted sequences contained sizeable gaps (Additional file 14), which could in part be due to the quality of the genome, as we indicated for our prediction of the B. mori Dscam- $h v$ gene (Additional file 2). Furthermore, there was a sizeable gap in the predicted sequence of T. castaneum Dscam3 (Additional file 14), the placement of which also does not match the evolutionary relationships among the organisms.

The Dscam2, Dscam3 and Dscam4 clades all had multiple insect representatives (holometabolous and hemimetabolous species) suggesting that they may have arisen before radiation occurred within the insects. However, Dscam 5 and Dscam6 contained representatives from only one hemimetabolous insect, P. humanus, and one holometabolous insect, $A$. mellifera: it seems unlikely that all of the other holometabolous insects have lost this gene. Our conservative cut-off for including putative genes in our phylogeny may have resulted in us missing representatives for these two clades from some of the other species. In the absence of topological constraints, but also when enforcing monophyly separately for each of the Dscam-hv and Dscam-like gene clades, the Dscam3 clade was basal to the rest of genes in the family (Additional files 22). Given the evolutionary relationships among arthropods (Figure 2), we hypothesised that the I. scapularis genes could be basal to those of the rest of the Pancrustacea. We tested this hypothesis by reconstructing a phylogeny in which I. scapularis Dscam co-orthologs were forced to be basal (Figure 3), the results of a SH-test showed that it was not significantly worse than the one in which the Dscam3 genes were basal (Additional file 15). We therefore propose that the ancestral Dscam gene was present in the genomes of the ancestral arthropods. The ancestral ortholog in the I. scapularis lineages underwent duplications and generated the Dscam I. scapularis coorthologs, while the ortholog in the ancestral pancrustaceans also underwent duplication events that developed the family further. The alternative hypothesis would be that the monophyly of the four Dscam I. scapularis inparalogs is an artifact caused by long-branch attraction and/or by convergence in amino acid preferences of the four genes in the I. scapularis genome. Only future research on basal arthropods will provide genomic data to test these exclusive hypotheses. Regarding crustaceans, the only gene prediction for the $D$. pulex genome corresponded to a Dscam-hv gene. This could be interpreted as the Dscam-hv clade to be basal to the rest of the Dscam-like ones. Indeed, a phylogenetic reconstruction enforcing such topology was not significantly worse than the best-known constrained tree, with Dscam3 being basal to the rest of pancrustacean Dscam clades (Additional file 15). The alternative hypothesis would either imply the selective loss of the Dscam-like gene in the $D$. pulex lineage, or the inability of our approach to detect it in its present form [see [10,73]].

To gain a deeper insight into the evolution of the Dscam-like and Dscam-hv clades, we dated our preferred tree using Bayesian reconstruction (Figure 4; see Additional file 24 for a reconstruction using the best tree). Our preferred dated tree where the $I$. scapularis clade was enforced as an outgroup (Figure 4) estimated that the I. scapularis clade split from the rest of the arthropods between 1020-688 million years ago (Ma), and the best tree estimate was between 928-642 Ma. These figures contain, but are on the edge of, Pisani's [75] 698.5 Ma mean divergence time estimate between Pancrustacea and Chelicerata. Our estimated date for the divergence of the deuterostomes and the protostomes (approximately $1000 \mathrm{Ma}$ ) is also quite old, but falls within other estimates, which range from 1,200 to $580 \mathrm{Ma}$ [76]. Within the Insecta, the most recent common ancestor (mrca) respectively for the main Dscam-like clades (Dscam2, Dscam3 and Dscam4) was between 919-638 Ma (Figure 4). Furthermore it is noteworthy that in the D. melanogaster genome, the Dscam 2 and the Dscam4 genes, closely related according to our reconstructions, are both encoded in chromosome $3 \mathrm{~L}$, ca. 1 mega base pairs apart from one each other. The two additional basal clades, Dscam5 and Dscam6, appeared to have arisen from more recent duplication events (Figure 4 and Additional file 24). Our estimate for the mrca of the Dscam-hv (D. pulex and L. vannamei: around 586-407 Ma; Figure 4) fits quite well with other molecular estimates for the time of divergence between these species (respectively, 508-533 Ma and $540 \mathrm{Ma}[77,78])$. Furthermore our estimates for the basal splits within the insects, i.e., Dscam-like genes from hemimetabolous and holometabolous insects, were in the region of another estimate (Figure 4, Additional file 24; e.g. $355 \mathrm{Ma}$ [30]).

More generally, our strict criteria for including a gene prediction as a putative Dscam ortholog (see methods), may have also resulted in us missing representatives from Dscam-like clades other than Dscam5 and Dscam6. For example, we identified no P. humanus Dscam3 and Dscam4 orthologs, and no Dscam4 ortholog for either A. mellifera or T. castaneum (Figure 2). It is possible that orthologs to those genes exist in the corresponding genomes, or alternatively, that after duplication events of the Dscam-like genes in the ancestor of the insects that these representatives were secondarily lost from some genomes. It is also unclear which mechanisms led to the generation of the copies of the ancestral Dscam 


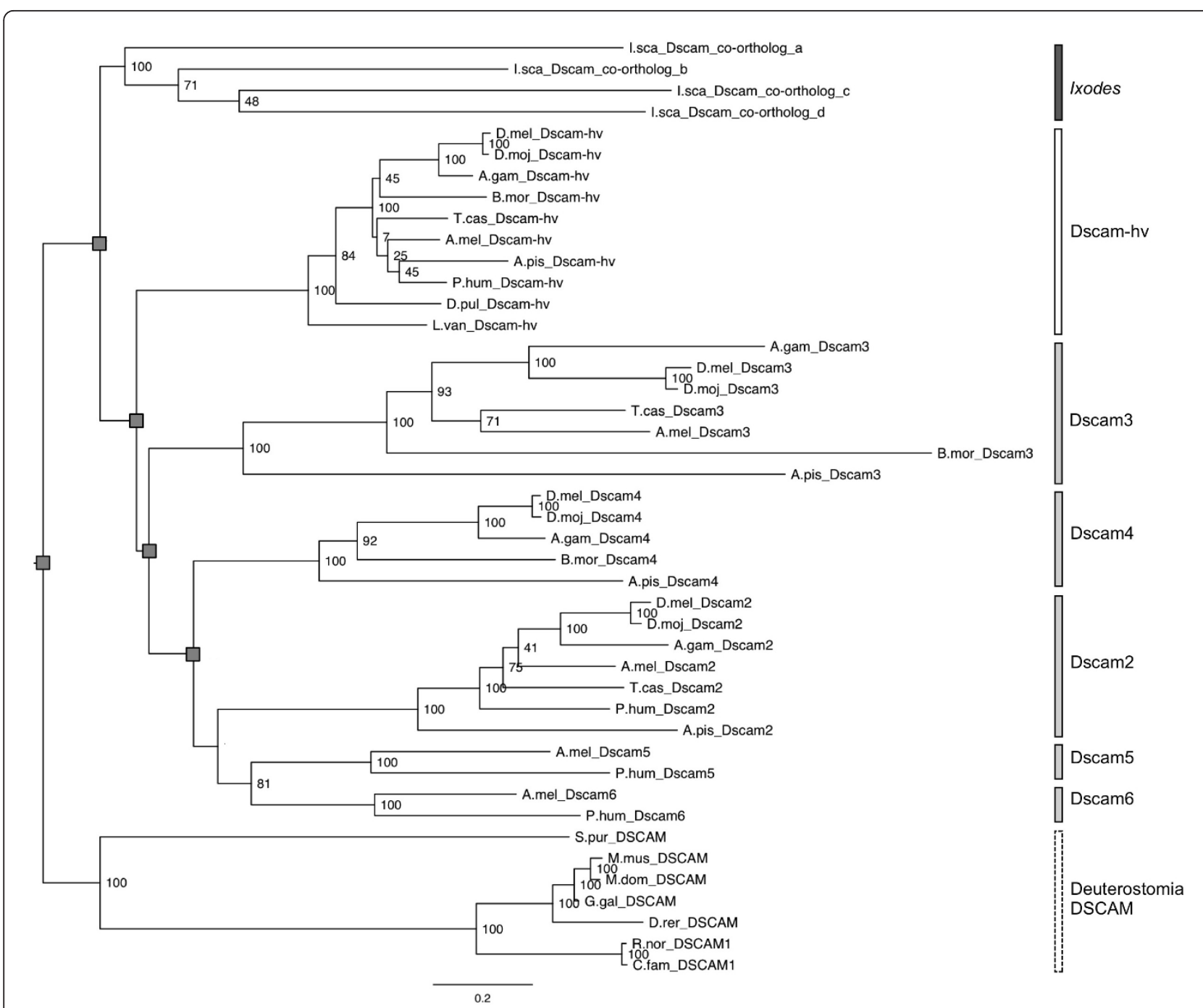

Figure 3 Maximum likelihood (RAXML) phylogeny of the Dscam/DSCAM gene family. Topology follows that of the preferred tree (Additional file 15), where grey squares indicate nodes that were fixed. Bootstrap values are shown at the non-fixed nodes. The vertical bars follow the bar colours of taxa written in black in Figure 2. The scale bar represents 0.2 substitutions per site.

gene in the genome of the ancestral insects in a relatively short period of time. It is indeed difficult to envisage how the duplication of a gene such as Dscam- $h v$, which spans almost 70 kilo base pairs in D. melanogaster, can be neutral and come at no cost. The conundrum about the forces driving the duplication and the advantages that condition the selection of the newly evolved genes make it difficult to classify these events according to the models of the evolution of gene duplications presented by Innan and Kondrashov [79].

\section{Identification and annotation of Dscam-hv hypervariable exons}

Our HMMs found identical numbers of alternatively spliced exons to species where exon numbers had previously been predicted or shown to be expressed (see
Additional file 2 for the three exceptions in Ig3). Using HMMs we annotated individual hypervariable exons from four insect species (Figure 2). For the four putative I. scapularis Dscam co-orthologs we did not find more than one Ig2, Ig3 or Ig7 ortholog within the regions of the genes searched. However, Dscam co-ortholog a appeared to have no Ig2 or Ig3 alternatively spliced variant (but see Additional file 2). We found no evidence of potential exon inversion events, i.e., putative exons were only found in three reading frames per genome.

\section{Phylogeny of Dscam-hv exons}

An especially interesting aspect of the evolution of the Dscam- $h v$ genes is the evolution of the alternatively spliced regions. In particular, is it possible to reconstruct how many alternatively spliced exons of each exon 


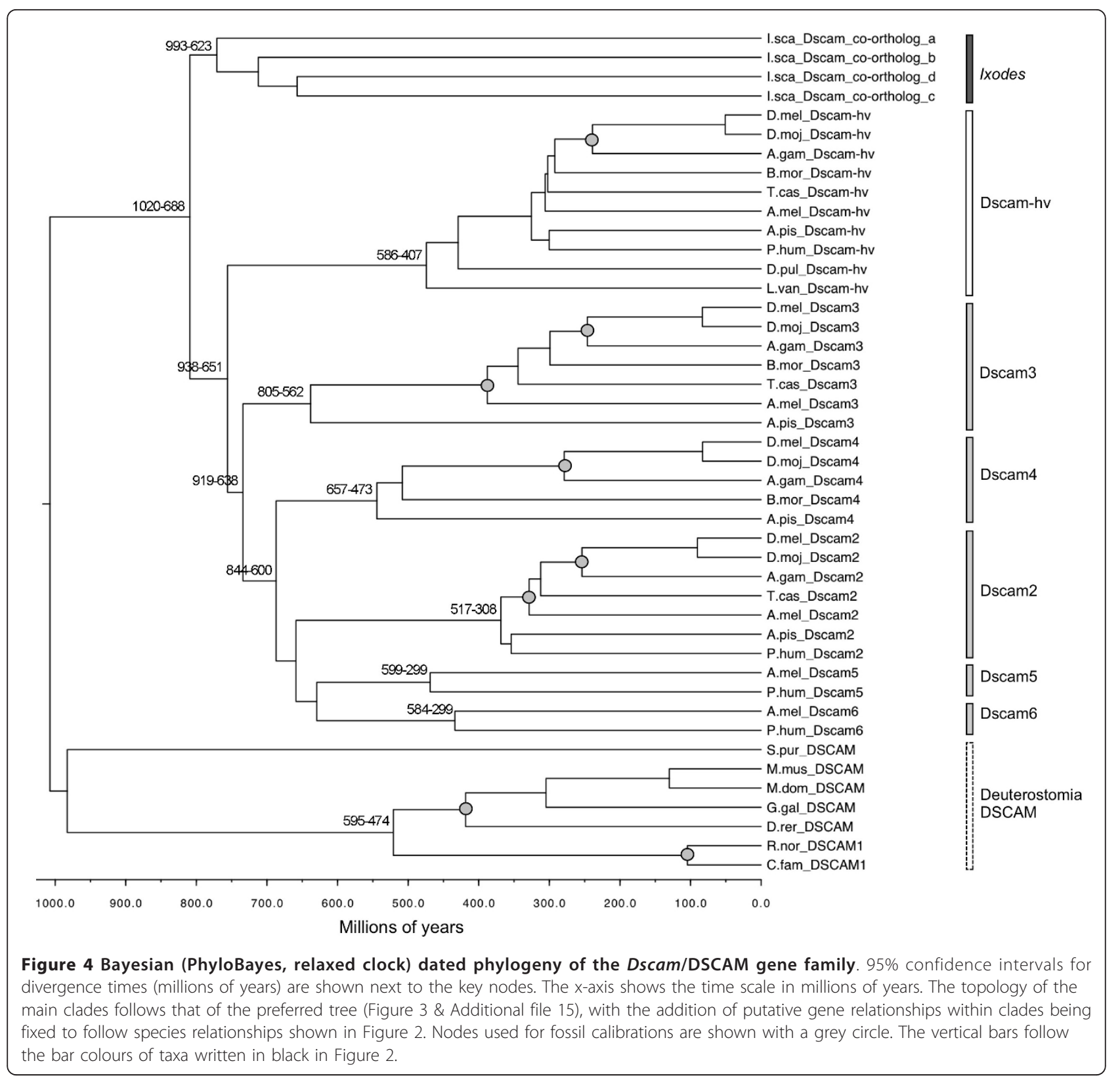

cluster were present at the base of the Pancrustacea and the Insecta, and did the three exon clusters evolved similarly? Using annotated exons from the literature and those predicted from our HMMs, we had 106 arthropod exons, ranging from 153 to 171 base pairs (bp) in length, with which to build the Ig2 phylogeny (Additional files 25 and 26). The phylogenetic relationships among exons could not be resolved with confidence, neither with maximum likelihood nor with Bayesian approaches (Additional files 25 and 26). In all cases the inferred trees displayed a star-like topology, without polarity, hindering the identification of groups of taxa or of exons that could belong together and making it unfortunately not possible to estimate the number of exon variants present at the base of the Arthropoda, Pancrustacea and Insecta. Indeed, for 58\% (72/106) of the sequences the position in the tree varied largely with the region analysed and showed multiple possible insertion points to reach an accumulated 0.95 posterior probability. This is perhaps not surprising, since we were trying to resolve the relationships among 106 sequences with 197 alignment patterns. The fact that we were unable to reconstruct the deep relationships between the exon variants with confidence differs from the findings of Lee and co-workers [80], where they reconstructed the evolutionary history of each variable 
exon cluster across holometabolous insects and a crustacean species (D. pulex) and subsequently suggested that there were at least nine Ig2 exons in the insect ancestor and one exon present in the common ancestor of Pancrustacea. Our low bootstrap support values and posterior probabilities (Additional files 25 and 26) are likely the result of the relatively short lengths of a large number of exons combined with a long evolutionary timescale. Additional factors such as accelerated evolutionary rate, subfunctionalisation and/or gene conversion, may have further contributed to the erosion of the phylogenetic signal. Finally, a similar composition across nonorthologous exons due to amino acid and/or codon usage preferences (e.g., [81]) might complicate the analysis, and generate a misleading impression of monophyly. Examples of this putative convergence can be observed, for example, in Additional files 25 and 26, where exons predicted in the same genome tend to flock together. One methodological explanation for the difference between our study and that of Lee and co-workers [80] is that we used Bayesian and maximum likelihood methods whereas they used neighbour joining. However, such high resolution power for neighbour joining compared to maximum likelihood and to Bayesian analysis is unexpected, especially for short, highly divergent sequences, as is the case with these sequences.

Because we could not infer the relationships between the exons over such an enormous evolutionary time scale, we tested whether we could find a better resolution at a scale of around 40-60 million years, i.e., the estimated divergence times for $D$. melanogaster and $D$. mojavensis $[82,83]$. Most exons from one species had clear orthologs in the other species (Figure 5 and Additional file 27), with no evolutionary events, whether losses or duplications, occurring since the species split. The number of rogue taxa decreased to $16 \%$ (4/25), but the position of the root and the fine relationships between orthologous pairs could not be resolved (Figure 5 and Additional file 27).

Our Ig3 phylogeny consisted of 290 arthropod exons, ranging from 102 to $132 \mathrm{bp}$ in length, and the Ig7 phylogeny consisted of 219 arthropod exons, ranging from 243 to $312 \mathrm{bp}$ in length. It was not possible to reconstruct a likely set of ancient exons for either of these exon clusters because of the low bootstrap support and posterior probabilities, and because a large proportion of the sequences were rogue taxa (64\% and 46\%, Ig3 and Ig7 respectively). Noticeably, many exons in the phylogeny are in species-specific clusters (Additional files 28, 29, 30). Again, it is difficult to say whether this clustering is due to multiple, parallel expansion events within a lineage, the current mainstream interpretation, because of the potentially confounding effect of codon usage variation across species [81] that may hinder

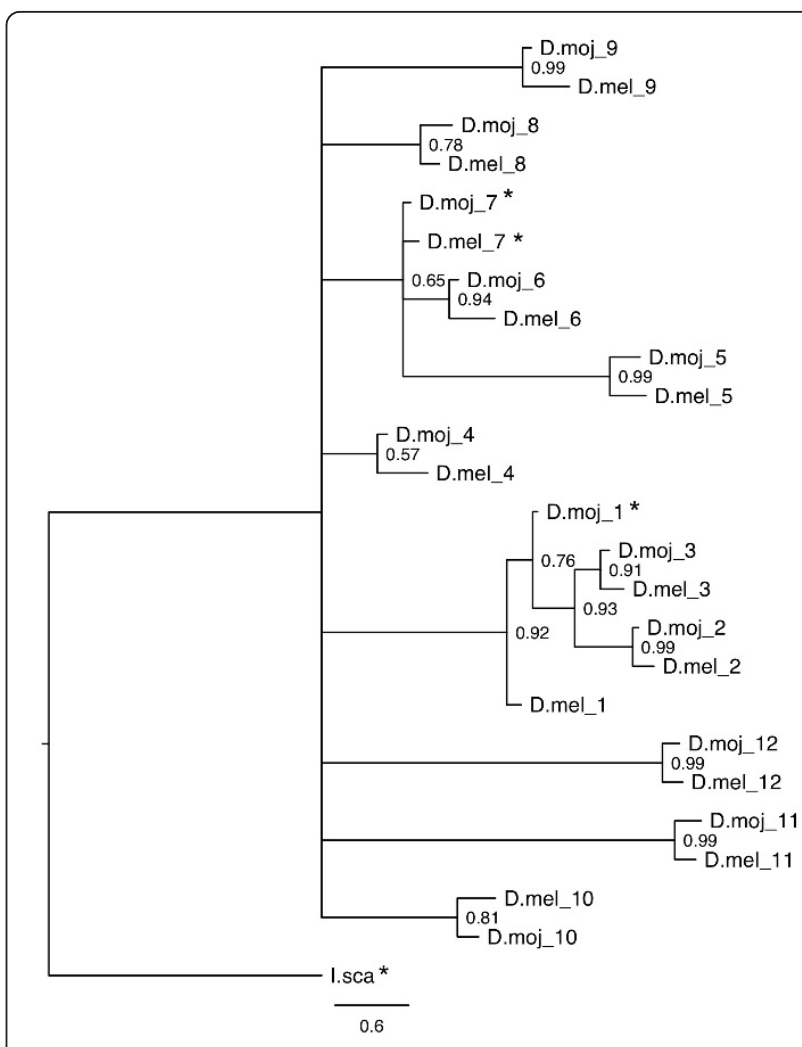

Figure 5 Bayesian (PhyloBayes) phylogeny of hypervariable lg2 variants (exon 4) from Drosophila melanogaster and $D$.

mojavensis. A putative Ixodes scapularis lg2 sequence was used as the outgroup. Bootstrap values are shown at the nodes. The scale bar represents 0.6 substitutions per site. Stars indicate rogue taxa, hence their phylogenetic positions cannot be inferred with confidence.

phylogenetic reconstruction. As mentioned above, the clusters may therefore reflect nucleotide composition and not necessarily common ancestry. Similarly, Lee and co-workers [80] suggested limited ancestral reconstruction was possible, although they suggested that six Ig3 exons and two Ig7 exons were present in the insect ancestor.

While the total number of Ig3 exon variants in $D$. melanogaster only differs by two from that of $D$. mojavensis, a closer look at the overall phylogeny (Additional files 32 and 33) reveals that there were probably more than two duplication or deletion events in these lineages. This is in contrast to the Ig2 phylogeny, and underlines the fact that the accumulation of diversity in the Ig3 cluster occurred faster than in the Ig2 cluster. The orthologous Ig7 exons identified in D. melanogaster and $D$. mojavensis were consistent with one another across the two phylogenetic reconstruction methods (Additional files 34 and 35), they showed that the first half of the alternatively spliced exon variants (9.1 to 9.14) corresponded almost one for one to orthologs in 
both species, the exons in the middle region show slightly more complex across-species relationships indicative of duplications and losses, and towards the end of the exon cluster D. melanogaster exons 9.27 to 9.33 and their $D$. mojavensis counterparts also appear to be clear orthologs of one another. However, it was still not possible to resolve the deeper relationships between the orthologs.

Overall, the three hypervariable exon clusters appear to have experienced quite different evolutionary histories. While the Ig2 cluster is comparatively more conserved (consistent with $[25,28]$ ), Ig3 and $\operatorname{Ig} 7$ have experienced many more recent exon duplications and deletions and most of the diversity appears to have evolved since the split of the insects. All exon clusters seem to have evolved mainly via tandem duplications, and possibly fall into category III 'Diversifying selection' according to the models of gene-duplication evolution presented by Innan and Kondrashov [79]. No duplications of more than one exon could be found in the Drosophila analysis. The three exon clusters have also evolved independently of one another. The differences between the three exon clusters regarding their evolution are likely caused by different selection pressures on the exons. These could be due to different functions, for example in the nervous system versus in the immune system, but also due to their different position in the three-dimensional structure of the Dscam protein [84].

\section{Conclusions}

Our analyses of the evolution of the Dscam family in the arthropods are compatible with the following evolutionary scenario. The genome of the arthropod ancestors already contained one copy of the ancestral Dscam gene. In the arachnid I. scapularis, gene duplication and diversification generated the four extant copies (coorthologs) of the Dscam gene, which are exclusive to this lineage. In the pancrustaceans, a basal duplication event generated the ancestral Dscam- $h v$ and the ancestor of the rest of the Dscam-like clades. The ancestral Dscam-like gene underwent a further series of between two and four duplication events in the lineage of the insects. In the Dscam- $h v$ lineage, the expansion of alternatively spliced exons in the Ig2, Ig3 and Ig7 domains via duplication started early, or immediately after, the emergence of the ancestral Dscam- $h v$ genes in pancrustaceans, and seems to be basal to this clade. There seems thus to exist an adaptive value in this broadening of the Dscam-hv transcript/protein repertoire that may have been available through neofunctionalisation after gene duplication. Such new function(s) could be related to the differentiation between self and non-self, as evidenced in their role in the nervous system and in their putative role in immunity. If it is true that the hypervariability in Dscam-hv arose after the split between arachnids and insects, one may wonder whether such new roles correspond to new solutions to old problems, also faced by arachnids and solved by other means, or whether they correspond to new functions that are exclusive to insects. A future search on more genomes with better quality will help fill the evolutionary gaps and clear the oddities that remain unexplained. Finally, only experimentation will enable us to solve questions about whether the putative Dscam-like genes are expressed and what their functions are, and to understand more about the role of alternative splicing of the hypervariable exons in Dscam- $h v$ across the arthropods.

\section{Additional material}

\section{Additional file 1: An overview of the workflow followed for the HMM construction and use, and for the gene predictions. \\ Additional file 2: Supplementary methods.}

Additional file 3: Genbank (NCBI) accession numbers for the known and putative Dscam-hv and Dscam-like genes used in the overall phylogenies.

Additional fie 4: Information regarding the HMMs for the Dscamlike gene search. Including the HMM identities, the lengths of each of the HMMs in amino acids, and the HMM amino acid start position relative to the D. melanogaster Dscam2 sequence (total length 2,040 aa).

\section{Additional file 5: Dscam-like HMM.}

Additional file 6: Putative Dscam-like orthologs/co-orthologs and the number of Hidden Markov Models positively identified for each ortholog/co-ortholog. Grey boxes indicate an HMM hit with an e-value $\leq 0.001$. White boxes indicate an e-value greater than 0.001 or no match. The identities of the Dscam-like genes were assigned according to the phylogenetic tree in Figure 3.

Additional file 7: D. mojavensis HMM results. Results for the Dscamlike HMMs built from A. mellifera, D. melanogaster and T. castaneum and run against the translated $D$. mojavensis genome. For a total of five 'matching' HMMs and above we found only four hits in the genome (i.e. four genes: one Dscam-hv and three Dscam-like), which had HMMs with significant hits in the correct order. The conservative cut-off value, which was subsequently used when searching other species for Dscam-like genes, is shown as a dashed line, i.e. a minimum of six HMMs had to match the sequence in the correct order and all with an e-value below 0.001 .

Additional file 8: Arthropod HMM results. Number of hits for the Dscam-like HMMs built from A. mellifera, D. melanogaster, D. mojavensis and $T$. castaneum and run against the translated genomes of A. gambiae, A. mellifera, A. pisum, B. mori, D. pulex, I. scapularis, P. humanus humanus and $T$. castaneum. The cut-off value is shown as a dashed line.

Additional file 9: Dscam-hv HMM.

Additional file 10: Ig2 HMM.

Additional file 11: $\lg 3$ HMM.

Additional file 12: $\lg 7$ HMM.

Additional file 13: E-value distribution among putative hypervariable exons found across all arthropod species using our HMMs. The vertical dashed line marks the cut-off e-value of 0.0001 .

Additional file 14: Amino acid alignment of putative Dscam gene family members. The complete protein alignment of 44 sequences was created using MUSCLE. All lg2, lg3 and lg7 orthologous regions were removed from the alignment. The resulting alignment was shortened using Gblocks. 
Additional file 15: Tests of alternative tree topologies. The "best" tree (top; Additional files 22 \&23) was tested against alternative hypotheses for the relationships between different Dscam clades by constructing alternative topologies (bottom two trees) and performing the Shimodaira-Hasegawa test. Neither of the two alternative topologies was significantly worse than the "best" tree at the $1 \%$ level.

Additional file 16: Nucleotide alignment of all arthropod Ig2 variants.

Additional file 17: Nucleotide alignment of all arthropod Ig3 variants.

Additional file 18: Nucleotide alignment of all arthropod Ig7 variants.

Additional file 19: Nucleotide alignment of all D. melanogaster and D. mojavensis Ig2 variants.

Additional file 20: Nucleotide alignment of all $D$. melanogaster and D. mojavensis lg3 variants.

Additional file 21: Nucleotide alignment of all $D$. melanogaster and D. mojavensis $\lg 7$ variants.

Additional file 22: Maximum likelihood (RAxML) phylogeny of the Dscam/DSCAM gene family, resulting in the best tree (Additional files 15 and 23). Bootstrap values (out of 100) are shown at the nodes. The vertical bars to the right are the same as in Figures 3 and 4 and follow the taxa colour codes in Figure 2. The scale bar represents 0.2 substitutions per site.

Additional file 23: Bayesian (PhyloBayes) phylogeny of the Dscam/ DSCAM gene family, resulting in the best tree (Additional file 15 \& Additional file 22). Posterior probabilities are shown at the nodes. The vertical bars to the right are the same as in Figures 3 and 4 and follow the taxa colour codes in Figure 2. The scale bar represents 0.4 substitutions per site.

Additional file 24: Bayesian (PhyloBayes, relaxed clock) dated phylogeny of the Dscam/DSCAM gene family. $95 \%$ confidence intervals for divergence times (millions of years) are shown next to the key nodes. The $x$-axis shows the time scale in millions of years. The topology follows that of the original best tree (see Additional file 15, 22 and Additional file 23). Nodes used for fossil calibrations are shown with a grey circle, for details see materials and methods. The vertical bars follow the bar colours of taxa written in black in Figure 2.

Additional file 25: Bayesian (PhyloBayes) phylogeny of all hypervariable Ig2 variants across the arthropods. A putative lxodes scapularis lg2 sequence is the outgroup. Bootstrap values are shown at the nodes. The scale bar represents 0.4 substitutions per site.

Additional file 26: Maxiumum likelihood (RAxML) phylogeny of all hypervariable lg2 variants across the arthropods. A putative Ixodes scapularis $\lg 2$ sequence is the outgroup. Bootstrap values are shown at the nodes. The scale bar represents 0.3 substitutions per site.

Additional file 27: Maximum likelihood (RAxML) phylogeny of hypervariable Ig2 variants (exon 4) from Drosophila melanogaster and $D$. mojavensis. A putative lxodes scapularis lg2 sequence is the outgroup. Bootstrap values are shown at the nodes. The scale bar represents 0.3 substitutions per site.

Additional file 28: Bayesian (PhyloBayes) phylogeny of all hypervariable Ig3 variants across the arthropods. A putative Ixodes scapularis lg3 sequence is the outgroup. Bootstrap values are shown at the nodes. The scale bar represents 0.3 substitutions per site.

Additional file 29: Maxiumum likelihood (RAxML) phylogeny of all hypervariable Ig3 variants across the arthropods. A putative Ixodes scapularis $\lg 3$ sequence is the outgroup. Bootstrap values are shown at the nodes. The scale bar represents 0.4 substitutions per site.

Additional file 30: Bayesian (PhyloBayes) phylogeny of all hypervariable Ig7 variants across the arthropods. A putative Ixodes scapularis lg7 sequence is the outgroup. Bootstrap values are shown at the nodes. The scale bar represents 0.5 substitutions per site.

Additional file 31: Maxiumum likelihood (RAxML) phylogeny of all Ig7 variants across the arthropods. A putative lxodes scapularis lg7 sequence is the outgroup. Bootstrap values are shown at the nodes. The scale bar represents 0.6 substitutions per site.

Additional file 32: Bayesian (PhyloBayes) phylogeny of hypervariable Ig 3 variants (exon 6) from Drosophila melanogaster and $D$. mojavensis. A putative Ixodes scapularis $\lg 3$ sequence is the outgroup. Bootstrap values are shown at the nodes. The scale bar represents 1 substitution per site.

Additional file 33: Maximum likelihood (RAxML) phylogeny of hypervariable Ig3 variants (exon 6) from Drosophila melanogaster and $D$. mojavensis. A putative /xodes scapularis lg3 sequence is the outgroup. Bootstrap values are shown at the nodes. The scale bar represents 0.6 substitutions per site.

Additional file 34: Bayesian (PhyloBayes) phylogeny of hypervariable Ig7 variants (exon 9) from Drosophila melanogaster and $D$. mojavensis. A putative Ixodes scapularis lg7 sequence is the outgroup. Bootstrap values are shown at the nodes. The scale bar represents 2 substitutions per site.

Additional file 35: Maximum likelihood (RAxML) phylogeny of hypervariable Ig7 variants (exon 9) from Drosophila melanogaster and $D$. mojavensis. A putative lxodes scapularis lg7 sequence is the outgroup. Bootstrap values are shown at the nodes. The scale bar represents 0.3 substitutions per site.

\section{Acknowledgements}

We would like to thank Daniela Brites for access to unpublished work. This work was supported by a grant from the Volkswagen Stiftung for SAOA (grant number: 1/83 516) and for IGB (recipient of a professorship under the Volkswagen Stiftung program "Evolutionary Biology"), and by the late Spanish Ministry for Science and Innovation (Programa Ramón y Cajal and grants BFU2009-06702-E/BMC and CGL2010-16713) to IGB.

\section{Author details}

${ }^{1}$ Institute for Evolution and Biodiversity, University of Münster, Hüfferstrasse 1, 48149 Münster, Germany. ${ }^{2}$ Unit of Infections and Cancer, Catalan Institute of Oncology (ICO), Gran Via de L' Hospitalet, 199, 08907 L'Hospitalet de Llobregat, Barcelona, Spain.

\section{Authors' contributions}

SAOA participated in the conception and design of the study, participated in analyses and drafted the manuscript. RF built the HMMs, identified the putative Dscam sequences, and participated in building the phylogenetic trees. JK participated in the conception of the study and helped to draft the manuscript. IGB participated in the conception and design of the study, performed the phylogenetic and statistical analyses, and helped to draft the manuscript. All authors read and approved the manuscript

Received: 14 November 2011 Accepted: 13 April 2012

Published: 13 April 2012

\section{References}

1. C. elegans Sequencing Consortium: Genome Sequence of the Nematode C. elegans: A Platform for Investigating Biology. Science 1998, 282:2012-2018.

2. International Human Genome Sequencing Consortium: Finishing the euchromatic sequence of the human genome. Nature 2004, 431:931-945.

3. Black DL: Mechanisms of alternative pre-messenger RNA splicing. Annu Rev Biochem 2003, 72:291-336.

4. Blencowe BJ: Alternative splicing: new insights from global analyses. Cell 2006, 126(1):37-47

5. Nilsen TW, Graveley BR: Expansion of the eukaryotic proteome by alternative splicing. Nature 2010, 463:457-463.

6. Schmucker D, Clemens JC, Shu H, Worby CA, Xiao J, Muda M, Dixon JE, Zipursky SL: Drosophila Dscam is an axon guidance receptor exhibiting extraordinary molecular diversity. Cell 2000, 101:671-684.

7. Wang J, Ma X, Yang JS, Zheng X, Zugates CT, Lee C-HJ, Lee T: Transmembrane/juxtamembrane domain-dependent Dscam distribution 
and function during mushroom body neuronal morphogenesis. Neuron 2004, 43:663-672.

8. Yu H-H, Yang JS, Wang J, Huang Y, Lee T: Endodomain diversity in the Drosophila Dscam and its roles in neuronal morphogenesis. J Neurosci 2009, 29:1904-1914.

9. Adams MD, Celniker SE, Holt RA, Evans CA, Gocayne JD, Amanatides PG, Scherer SE, Li PW, Hoskins RA, Galle RF, et al: The Genome Sequence of Drosophila melanogaster. Science 2000, 287:2185-2195.

10. Brites D, McTaggart S, Morris K, Anderson J, Thomas K, Colson I, Fabbro T, Little TJ, Ebert D, Du Pasquier L: The Dscam homologue of the crustacean Daphnia is diversified by alternative splicing like in insects. Mol Biol Evol 2008, 25:1429-1439.

11. Schmucker D, Chen B: Dscam and DSCAM: complex genes in simple animals, complex animals yet simple genes. Genes Dev 2009, 23:147-156.

12. Hummel T, Vasconcelos ML, Clemens JC, Fishilevich Y, Vosshall LB, Zipursky SL: Axonal targeting of olfactory receptor neurons in Drosophila is controlled by Dscam. Neuron 2003, 37:221-231.

13. Hattori D, Demir E, Kim HW, Viragh E, Zipursky SL, Dickson BJ: Dscam diversity is essential for neuronal wiring and self-recognition. Nature 2007, 449:223-227.

14. Matthews BJ, Kim ME, Flanagan JJ, Hattori D, Clemens JC, Zipursky SL, Grueber WB: Dendrite self-avoidance is controlled by Dscam. Cell 2007, 129:593-604.

15. Wojtowicz WM, Wu W, Andre I, Qian B, Baker D, Zipursky SL: A vast repertoire of Dscam binding specificities arises from modular interactions of variable Ig domains. Cell 2007, 130:1134-1145.

16. Hattori D, Chen Y, Matthews BJ, Salwinski L, Sabatti C, Grueber WB, Zipursky SL: Robust discrimination between self and non-self neurites requires thousands of Dscam1 isoforms. Nature 2009, 461:644-648.

17. Watson FL, Püttmann-Holgado R, Thomas F, Lamar DL, Hughes M, Kondo M, Rebel VI, Schmucker D: Extensive diversity of Ig-superfamily proteins in the immune system of insects. Science 2005, 309:1874-1878.

18. Dong Y, Taylor HE, Dimopoulos G: AgDscam, a hypervariable immunoglobulin domain-containing receptor of the Anopheles gambiae innate immune system. PLoS Biol 2006, 4:e229.

19. Smith P, Mwangi J, Afrane Y, Yan G, Obbard D, Ranford-Cartwright L, Little T: Alternative splicing of the Anopheles gambiae Dscam gene in diverse Plasmodium falciparum infections. Malar J 2011, 10:156.

20. Watthanasurorot A, Jiravanichpaisal P, Liu H, Söderhäll I, Söderhäll K: Bacteria-Induced Dscam Isoforms of the Crustacean, Pacifastacus leniusculus. PLoS Pathog 2011, 7:1-14.

21. Boehm T: Two in one: dual function of an invertebrate antigen receptor. Nat Immunol 2007, 8:1031-1033.

22. Kurtz J, Armitage SAO: Alternative adaptive immunity in invertebrates. Trends Immunol 2006, 27:493-496.

23. Du Pasquier L: Insects diversify one molecule to serve two systems. Science 2005, 309:1826-1827.

24. Meijers R, Püttmann-Holgado R, Skiniotis G, J-h L, Walz T, J-h W Schmucker D: Structural basis of Dscam isoform specificity. Nature 2007, 449:487-491.

25. Graveley BR, Kaur A, Gunning D, Zipursky SL, Rowen L, Clemens JC: The organization and evolution of the dipteran and hymenopteran Down syndrome cell adhesion molecule (Dscam) genes. RNA 2004, 10:1499-1506.

26. Chou P-H, Chang H-S, Chen IT, Lin H-Y, Chen Y-M, Yang H-L, Wang KCH-C: The putative invertebrate adaptive immune protein Litopenaeus vannamei Dscam (LvDscam) is the first reported Dscam to lack a transmembrane domain and cytoplasmic tail. Dev Comp Immunol 2009, 33:1258-1267.

27. Chou P-H, Chang H-S, Chen IT, Lee C-W, Hung H-Y, Han-Ching Wang KC: Penaeus monodon Dscam (PmDscam) has a highly diverse cytoplasmic tail and is the first membrane-bound shrimp Dscam to be reported. Fish Shellfish Immunol 2011, 30:1109-1123.

28. Crayton ME, Powell BC, Vision TJ, Giddings MC: Tracking the evolution of alternatively spliced exons within the Dscam family. BMC Evol Biol 2006, 6:16-16.

29. Benton MJ, Donoghue PCJ: Paleontological evidence to date the tree of life. Mol Biol Evol 2007, 24:26-53.

30. Wiegmann BM, Trautwein MD, Kim J-W, Cassel BK, Bertone MA, Winterton SL, Yeates DK: Single-copy nuclear genes resolve the phylogeny of the holometabolous insects. BMC Biol 2009, 7:34-34.
31. Regier JC, Shultz JW, Kambic RE: Pancrustacean phylogeny: hexapods are terrestrial crustaceans and maxillopods are not monophyletic. Proc $R$ Soc Lond B Biol Sci 2005, 272:395-401.

32. JI S, Tautz D, Richards S, Weinstock GM, Gibbs RA, Werren JH, Tettelin H, Lercher MJ: Phylogenomic analysis reveals bees and wasps (Hymenoptera) at the base of the radiation of Holometabolous insects. Genome Res 2006, 16:1334-1338.

33. Peterson K, Cotton JA, Gehling JG, Pisani D: The Ediacaran emergence of bilaterians: congruence between the genetic and the geological fossil records. Philos Trans R Soc Lond B Biol Sci 2008, 363:1435-1443.

34. Jenner R: Higher-level crustacean phylogeny: consensus and conflicting hypotheses. Arthropod Struct Dev 2010, 39:143-153.

35. Grimaldi D, Engel MS: Evolution of the Insects New York: Cambridge University Press; 2005.

36. Vogel C, Teichmann SA, Chothia C: The immunoglobulin superfamily in Drosophila melanogaster and Caenorhabditis elegans and the evolution of complexity. Development 2003, 130:6317-6328.

37. Millard SS, Flanagan JJ, Pappu KS, Wu W, Zipursky SL: Dscam2 mediates axonal tiling in the Drosophila visual system. Nature 2007, 447:720-724.

38. Andrews GL, Tanglao S, Farmer WT, Morin S, Brotman S, Berberoglu MA, Price H, Fernandez GC, Mastick GS, Charron F, et al: Dscam guides embryonic axons by Netrin-dependent and -independent functions. Development 2008, 135:3839-3848.

39. Funada M, Hara H, Sasagawa H, Kitagawa Y, Kadowaki T: A honey bee Dscam family member, AbsCAM, is a brain-specific cell adhesion molecule with the neurite outgrowth activity which influences neuronal wiring during development. Eur J Neurosci 2007, 25:168-180.

40. Yamakawa K, Huot YK, Haendelt MA, Hubert R, Chen XN, Lyons GE, Korenberg JR: DSCAM: a novel member of the immunoglobulin superfamily maps in a Down syndrome region and is involved in the development of the nervous system. Hum Mol Genet 1998, 7:227-237.

41. Liu G, Li W, Wang L, Kar A, Guan K-L, Rao Y, Wu JY: DSCAM functions as a netrin receptor in commissural axon pathfinding. Proc Natl Acad Sci USA 2009, 106:2951-2956.

42. Fuerst PG, Bruce F, Tian M, Wei W, Elstrott J, Feller MB, Erskine L, Singer JH, Burgess RW: DSCAM and DSCAML1 function in self-avoidance in multiple cell types in the developing mouse retina. Neuron 2009, 64:484-497.

43. Agarwala KL, Ganesh S, Tsutsumi Y, Suzuki T, Amano K, Yamakawa K: Cloning and functional characterization of DSCAML1, a novel DSCAMlike cell adhesion molecule that mediates homophilic intercellular adhesion. Biochem Biophys Res Commun 2001, 285:760-772.

44. Eddy SR: Profile hidden Markov models. Bioinformatics 1998, 14:755-755.

45. Eddy SR: What is a hidden Markov model? Nat Biotechnol 2004, 22:1315-1316.

46. Wall D, Fraser H: AE H: Detecting putative orthologs. Bioinformatics 2003, 19:1710-1711.

47. Eddy SR: Hidden Markov models. Curr Opin Struct Biol 1996, 6:361-365.

48. Eddy SR: Accelerated Profile HMM Searches. PLoS Comput Biol 2011, 7: e1002195.

49. Chapman JA, Kirkness EF, Simakov O, Hampson SE, Mitros T, Weinmaier T, Rattei T, Balasubramanian PG, Borman J, Busam D, et al: The dynamic genome of Hydra. Nature 2010, 464:592-596.

50. Putnam NH, Srivastava M, Hellsten U, Dirks B, Chapman J, Salamov A, Terry A, Shapiro H, Lindquist E, Kapitonov W, et al: Sea anemone genome reveals ancestral eumetazoan gene repertoire and genomic organization. Science 2007, 317:86-94.

51. Pagel Van Zee J, Geraci NS, Guerrero FD, Wikel SK, Stuart JJ, Nene VM, Hill CA: Tick genomics: the Ixodes genome project and beyond. Int J Parasitol 2007, 37(12):1297-1305.

52. Colbourne JK, Pfrender ME, Gilbert D, Thomas WK, Tucker A, Oakley TH, Tokishita S, Aerts A, Arnold GJ, Basu MK, et al: The Ecoresponsive Genome of Daphnia pulex. Science 2011, 331:555-561.

53. Holt RA, Subramanian GM, Halpern A, Sutton GG, Charlab R, Nusskern DR, Wincker P, Clark AG, Ribeiro JMC, Wides R, et al: The genome sequence of the malaria mosquito Anopheles gambiae. Science 2002, 298:129-149.

54. Weinstock GM, Robinson GE, Gibbs RA, Worley KC, Evans JD, Maleszka R, Robertson HM, Weaver DB, Beye M, Bork P, et al: Insights into social insects from the genome of the honeybee Apis mellifera. Nature 2006, 443:931-949. 
55. The International Silkworm Genome Consortium: The genome of a lepidopteran model insect, the silkworm Bombyx mori. Insect Biochem Mol Biol 2008, 38:1036-1045.

56. Clark AG, Eisen MB, Smith DR, Bergman CM, Oliver B, Markow TA, Kaufman TC, Kellis M, Gelbart W, lyer VN, et al: Evolution of genes and genomes on the Drosophila phylogeny. Nature 2007, 450:203-218.

57. Richards S, Gibbs RA, Weinstock GM, Brown SJ, Denell R, Beeman RW Gibbs R, Bucher G, Friedrich M, Grimmelikhuijzen CJP, et al: The genome of the model beetle and pest Tribolium castaneum. Nature 2008 452:949-955.

58. The International Aphid Genomics Consortium: Genome sequence of the pea aphid Acyrthosiphon pisum. PLoS Biology 2010, 8:e1000313.

59. Kirkness EF, Haas BJ, Sun W, Braig HR, Perotti MA, Clark JM, Lee SH, Robertson HM, Kennedy RC, Elhaik E, et al: Genome sequences of the human body louse and its primary endosymbiont provide insights into the permanent parasitic lifestyle. Proc Natl Acad Sci USA 2010, 107:12168-12173.

60. Edgar RC: MUSCLE: multiple sequence alignment with high accuracy and high throughput. Nucleic Acids Res 2004, 32:1792-1797.

61. Salamov AA, Solovyev W: Ab initio Gene Finding in Drosophila Genomic DNA. Genome Res 2000, 10:516-522

62. Talavera G, Castresana J: Improvement of phylogenies after removing divergent and ambiguously aligned blocks from protein sequence alignments. Syst Biol 2007, 56:564-577.

63. Gribaldo S, Philippe H: Ancient Phylogenetic Relationships. Theor Popul Biol 2002, 61:391-408.

64. Le SQ, Gascuel O: An improved general amino acid replacement matrix. Mol Biol Evol 2008, 25:1307-1320.

65. Abascal F, Zardoya R, Posada D: ProtTest: selection of best-fit models of protein evolution. Bioinformatics 2005, 21:2104-2105.

66. Stamatakis A: RAxML-VI-HPC: maximum likelihood-based phylogenetic analyses with thousands of taxa and mixed models. Bioinformatics 2006 22:2688-2690

67. Lartillot N, Philippe H: A Bayesian mixture model for across-site heterogeneities in the amino-acid replacement process. Mol Biol Evol 2004, 21:1095-1109.

68. Pattengale ND, Aberer AJ, Swenson KM, Stamatakis A, Moret BME: Uncovering hidden phylogenetic consensus in large data sets. IEEE/ACM Trans Comput Biol Bioinform 2011, 8:902-911

69. Shimodaira H, Hasegawa M: Multiple Comparisons of Log-Likelihoods with Applications to Phylogenetic Inference. Mol Biol Evol 1999, 16:1114-1116

70. Thorne $J \mathrm{~L}$, Kishino $\mathrm{H}$, Painter IS: Estimating the rate of evolution of the rate of molecular evolution. Mol Biol Evol 1998, 15:1647-1657.

71. Suyama $M$, Torrents $D$, Bork P: PAL2NAL: robust conversion of protein sequence alignments into the corresponding codon alignments. Nucleic Acids Res 2006, 34:W609-612

72. Irimia M, Maeso I, Gunning PW, Garcia-Fernàndez J, Roy SW: Internal and external paralogy in the evolution of tropomyosin genes in metazoans. Mol Biol Evol 2010, 27:1504-1517.

73. Brites D, Encinas-Viso F, Ebert D, Du Pasquier L, Haag CR: Population genetics of duplicated alternatively spliced exons of the Dscam gene in Daphnia and Drosophila. PLoS One 2011, 6:e27947.

74. Sonnhammer ELL, Koonin EV: Orthology, parology and proposed classification for paralog subtypes. Trends Genet 2002, 18:619-620.

75. Pisani D: Arthropods (Arthropoda). In The timetree of life. Edited by: Blair Hedges S, Sudhir Kumar. Oxford: Oxford University Press; 2009:251-254.

76. Blair J: Animals (Metazoa). In The timetree of life. Edited by: Blair Hedges S, Sudhir Kumar. Oxford: Oxford University Press; 2009:223-230.

77. Gaunt MW, Miles MA: An insect molecular clock dates the origin of the insects and accords with palaeontological and biogeographic landmarks. Mol Biol Evol 2002, 19:748-761.

78. Otsuka J, Sugaya N: Advanced formulation of base pair changes in the stem regions of ribosomal RNAs; its application to mitochondrial rRNAs for resolving the phylogeny of animals. J Theor Biol 2003, 222:447-460.

79. Innan H, Kondrashov F: The evolution of gene duplications: classifying and distinguishing between models. Nat Rev Genet 2010, 11:97-108.

80. Lee C, Kim N, Roy M, Graveley BR: Massive expansions of Dscam splicing diversity via staggered homologous recombination during arthropod evolution. RNA 2009, 16:91-105.
81. Powell JR, Moriyama EN: Evolution of codon usage bias in Drosophila. Proc Natl Acad Sci USA 1997, 94:7784-7790.

82. Tamura K, Subramanian S, Kumar S: Temporal patterns of fruit fly (Drosophila) evolution revealed by mutation clocks. Mol Biol Evol 2004 21:36-44.

83. Russo CA, Takezaki N, Nei M: Molecular phylogeny and divergence times of drosophilid species. Mol Biol Evol 1995, 12:391-404.

84. Sawaya MR, Wojtowicz WM, Andre I, Qian B, Wu W, Baker D, Eisenberg D, Zipursky SL: A double S shape provides the structural basis for the extraordinary binding specificity of Dscam isoforms. Cell 2008, 134:1007-1018.

doi:10.1186/1471-2148-12-53

Cite this article as: Armitage et al:: The evolution of Dscam genes across the arthropods. BMC Evolutionary Biology 2012 12:53.

\section{Submit your next manuscript to BioMed Central and take full advantage of:}

- Convenient online submission

- Thorough peer review

- No space constraints or color figure charges

- Immediate publication on acceptance

- Inclusion in PubMed, CAS, Scopus and Google Scholar

- Research which is freely available for redistribution

Submit your manuscript at www.biomedcentral.com/submit
C Biomed Central 